\title{
Comparisons between ticagrelor and clopidogrel following percutaneous coronary intervention in patients with acute coronary syndrome: a comprehensive meta-analysis
}

This article was published in the following Dove Medical Press journal:

Drug Design, Development and Therapy

\author{
Zhong-Guo Fan' \\ Wen-Ling Zhang ${ }^{2}$ \\ Bing $X u^{\prime}$ \\ Jun Ji' \\ Nai-Liang Tian ${ }^{3}$ \\ Sheng-Hu He \\ 'Department of Cardiology, Subei \\ People's Hospital of Jiangsu Province, \\ Yangzhou University, Yangzhou, \\ Jiangsu, China; ${ }^{2}$ Department of \\ Geriatric Gastroenterology, First \\ Affiliated Hospital, Nanjing Medical \\ University, Nanjing, Jiangsu, China; \\ ${ }^{3}$ Department of Cardiology, Nanjing \\ First Hospital, Nanjing Heart Center, \\ Nanjing Medical University, Nanjing, \\ Jiangsu, China
}

\begin{abstract}
Background: The efficacy and safety of ticagrelor following percutaneous coronary intervention for patients with acute coronary syndrome remains unclear. This study sought to evaluate clinical outcomes of ticagrelor as part of dual-antiplatelet treatment for these patients.

Methods: PubMed, MEDLINE, Embase, and other Internet sources were searched for eligible citations. The primary end point was major adverse cardiovascular and cerebrovascular events, consisting of cardiovascular death, myocardial infarction, and stroke. The secondary end point was the occurrence of definite/probable stent thrombosis (ST). The risk of bleeding was chosen to be the safety end point.

Results: Eleven clinical trials - six randomized trials and five observational trials - were finally analyzed. A tendency toward reduction in the risk of major adverse cardiovascular and cerebrovascular events was observed only with respect to ticagrelor (OR 0.83, 95\% CI 0.66-1.03; $P=0.091$ ), which might have resulted from the lower risk of cardiovascular death (OR 0.78 , 95\% CI $0.68-0.89 ; P<0.001)$. The overall incidence of ST differed significantly between the ticagrelor group and the clopidogrel group (OR $0.74,95 \%$ CI $0.59-0.93 ; P=0.009$ ), but the risk of bleeding, regardless of major or minor bleeding, increased significantly.

Conclusion: As part of dual-antiplatelet treatment following percutaneous coronary intervention, ticagrelor significantly reduced the risk of cardiovascular death and ST in acute coronary syndrome patients, but at the cost of bleeding. More powerful relevant randomized trials are still warranted to guide clinical decision-making.
\end{abstract}

Keywords: ticagrelor, clopidogrel, dual antiplatelet treatment, percutaneous coronary intervention, acute coronary syndrome

\section{Introduction}

Patients with acute coronary syndrome (ACS) are at higher risk of mortality, especially those suffering from acute myocardial infarction (AMI), which commonly appears as a large amount of necrotic myocardium, making it easier for severe complications to occur. ${ }^{1}$ Since the rapidly developed interventional techniques that have been widely applied in clinic, improved clinical outcomes have been reported. ${ }^{2}$ According to the 2018 European Society of Cardiology (ESC)-European Association for CardioThoracic Surgery (EACTS) guidelines, percutaneous coronary intervention (PCI) was strongly recommended for patients with high-risk ACS (class I). ${ }^{3}$ Of note, a successful PCI procedure would strengthen these benefits, as well as routine dual-antiplatelet treatment (DAPT) consisting of aspirin and a P2Y12-receptor antagonist (clopidogrel), ${ }^{4}$ 
which might prevent the subsequence of stent thrombosis (ST) or restenosis mainly resulting from the superimposition of a platelet-rich thrombus. ${ }^{5}$ In fact, though routine DAPT following PCI had been widely applied, ST incidence is still high among these patients, because it remains difficult to overcome high on-treatment platelet reactivity (HTPR). ${ }^{6}$

Ticagrelor is a new, reversible, and direct-acting oral P2Y12 ADP-receptor antagonist, which has been reported to provide faster and greater inhibiting effects on platelet aggregation than clopidogrel. ${ }^{7,8}$ As such, it is preferred for patients with ACS, mainly based on results from the PLATO trial, indicating that significantly reduced risk of death from vascular causes, MI, or stroke is found with respect to ticagrelor. ${ }^{9}$ In contrast, another two large randomized controlled trials (RCTs) ${ }^{10,11}$ and two observational (Obs) trials ${ }^{12,13}$ showed no significant benefits of ticagrelor in reducing the occurrence of ischemic events among these patients. In addition, two recent comprehensive meta-analyses ${ }^{14,15}$ also reported antagonistic results of ticagrelor in such patients, but many interfering factors were involved, which might have limited analysis in terms of determining the real efficacy and safety of this new potent oral ADP-receptor antagonist. These conflicting data render the real efficacy and safety of ticagrelor controversial. As a result, we performed this comprehensive meta-analysis involving as many relevant clinical trials as possible to evaluate the efficacy and safety of ticagrelor following PCI for these patients.

\section{Methods}

\section{Literature search}

Electronic databases - PubMed, MEDLINE, Embase, and the Cochrane Controlled Trials Registry - were searched for eligible citations (last search was up to October 2018). To make sure all relevant studies would be included, combinations of several relevant keywords were used: "ticagrelor”, "clopidogrel”, “dual antiplatelet therapy", "DAPT", "acute myocardial infarction OR AMI", "acute coronary syndrome OR ACS", "percutaneous coronary intervention", "PCI" and "stent implantation". Additionally, possible relevant references from published review articles or meta-analyses were hand-searched for eligibility.

\section{Inclusion and exclusion criteria}

Eligible studies needed to meet inclusion criteria of adult patients suffering from ACS (age from 18 to 90 years) and undergoing PCI performed comparisons between ticagrelor and clopidogrel (routine maintenance dose $75 \mathrm{mg}$ ) as part of DAPT and follow-up $\geq 3$ months and reported results of adverse clinical events. Exclusion criteria were patients with cardiogenic shock or receiving oral anticoagulants (eg, warfarin, dabigatran, rivaroxaban), ongoing studies, duplicated studies, or different studies using the same sample, non-English-language studies, and review articles or meta-analyses.

\section{Data extraction, synthesis, and quality assessment}

Two independent investigators (ZGF and WLZ) reviewed all relevant citations using standardized data-abstraction forms to assess eligibility. Disagreements were resolved by the third investigator (BX). These extracted data from each included study were study name or authors of the trial, publication year, baseline demographics, characteristics of medical histories, and clinical outcomes during the follow-up. These trials were divided into two subgroups based on the study design: the RCT subgroup and the Obs subgroup. To assess the quality of each retrieved study, the Jadad score ${ }^{16}$ (for RCTs) or the Newcastle-Ottawa Scale ${ }^{17}$ (for Obs trials) was used as appropriate.

\section{Study end points}

The primary end point of this study was incidence of major adverse cardiovascular and cerebrovascular events (MACCEs), consisting of cardiovascular death (all-cause deaths were recorded instead in several trials, ${ }^{10,18,19}$ due to the absence of relevant data), MI, and stroke. The secondary end point was the risk of definite/probable ST, defined according to the Academic Research Consortium..$^{20}$ The occurrence of bleeding events, including major and minor bleeding, were chosen as the safety end points. There were slight differences with respect to definitions of clinical end points in these trials, and related data were recorded following the standardized definitions.

\section{Statistical analyses}

The meta-analysis was performed in accordance with the PRISMA (preferred reporting items for systematic reviews and meta-analysis) statement. ${ }^{21}$ All end points were recorded as dichotomous variables and relevant comparisons estimated with ORs and 95\% CIs. Stata 12.0 (StataCorp, College Station, TX, USA) was used for all statistical analyses, and all $P$-values were two-tailed. Statistical significance was considered when $P<0.05$. If the $P$-value of Cochrane's $Q$ test was $<0.10$ and/or the $I^{2}$ statistic was $\geq 50 \%$, significant heterogeneity was considered and a random-effect model 
subsequently selected. In contrast, a fixed-effect model with the Mantel-Haenszel method would be selected instead. Moreover, Egger's test was performed to assess publication bias and significant asymmetry considered if $P<0.1 .^{22}$ The stability of the treatment effects was evaluated via sensitivity analyses by excluding one study at a time.

\section{Results}

\section{Eligible studies and patient characteristics}

After screening of 539 initial articles through the electronic databases and another 29 from several other Internet sources, eleven clinical trials were finally enrolled: $\operatorname{six} \operatorname{RCTs}^{9-11,18,23,24}$ and five Obs trials ${ }^{12,13,19,25,26}$ (Figure 1). Among these Obs trials, three ${ }^{13,19,25}$ provided precise details and results for subgroups following propensity-score matching, while one study ${ }^{26}$ reported only propensity-score-matched clinical end points. We analyzed the data from these matched subgroups instead. The main baseline characteristics and dosing regimens of ticagrelor and clopidogrel of the trials are listed in Tables 1 and 2. Assessment of study qualities is described in Table 1 .

\section{Major adverse cardiovascular and cerebrovascular events}

There was no significant difference between the ticagrelor group and the clopidogrel group with respect to the overall risk of composite MACCEs (OR 0.83, 95\% CI 0.66-1.03, $P=0.091 ; I^{2}=49.5 \%, P=0.031$; Figure 2). Similarly, negative results were also shown in both the RCT (OR 0.79, 95\% CI 0.53-1.18, $\left.P=0.250 ; I^{2}=63.0 \%, P=0.019\right)$ and Obs subgroups (OR 0.82, 95\% CI 0.60-1.12, $P=0.221$; $\left.I^{2}=36.5 \%, P=0.031\right)$. Egger's test indicated no publication bias $(P=0.152)$.

\section{Cardiovascular death}

As shown in Figure 3, the overall incidence of cardiovascular death was significantly reduced by ticagrelor (OR 0.78 , 95\% CI 0.68-0.89, $\left.P<0.001 ; I^{2}=0, P=0.575\right)$, which might have been due to results of the RCT subgroup (OR 0.79 , 95\% CI $\left.0.68-0.90, P=0.001 ; I^{2}=8.8 \%, P=0.356\right)$. No publication bias was encountered on Egger's test $(P=1)$, and the superiority of ticagrelor was demonstrated by the relevant sensitivity analysis.

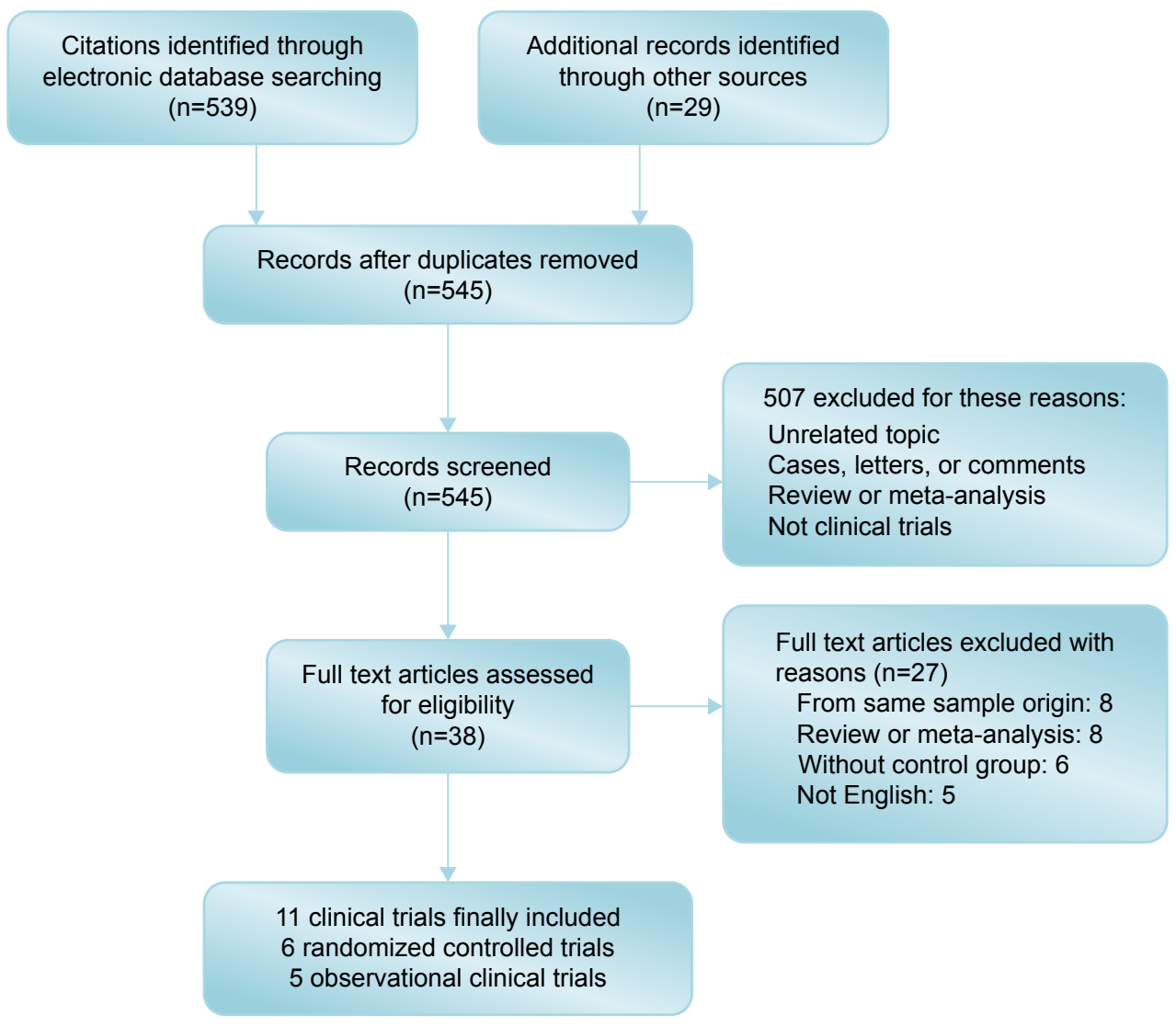

Figure I Flowchart depicting selection of studies included in this meta-analysis. 

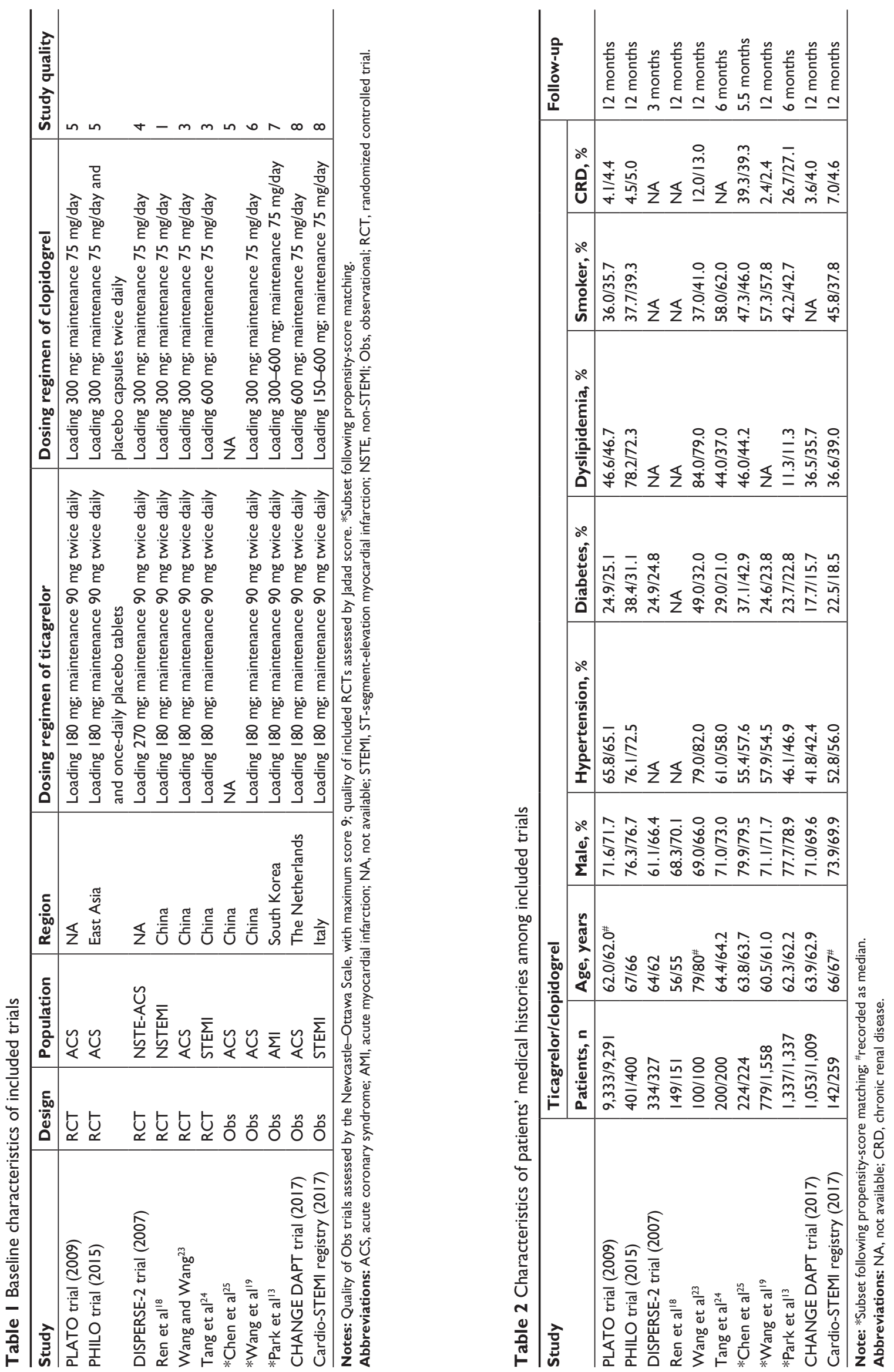


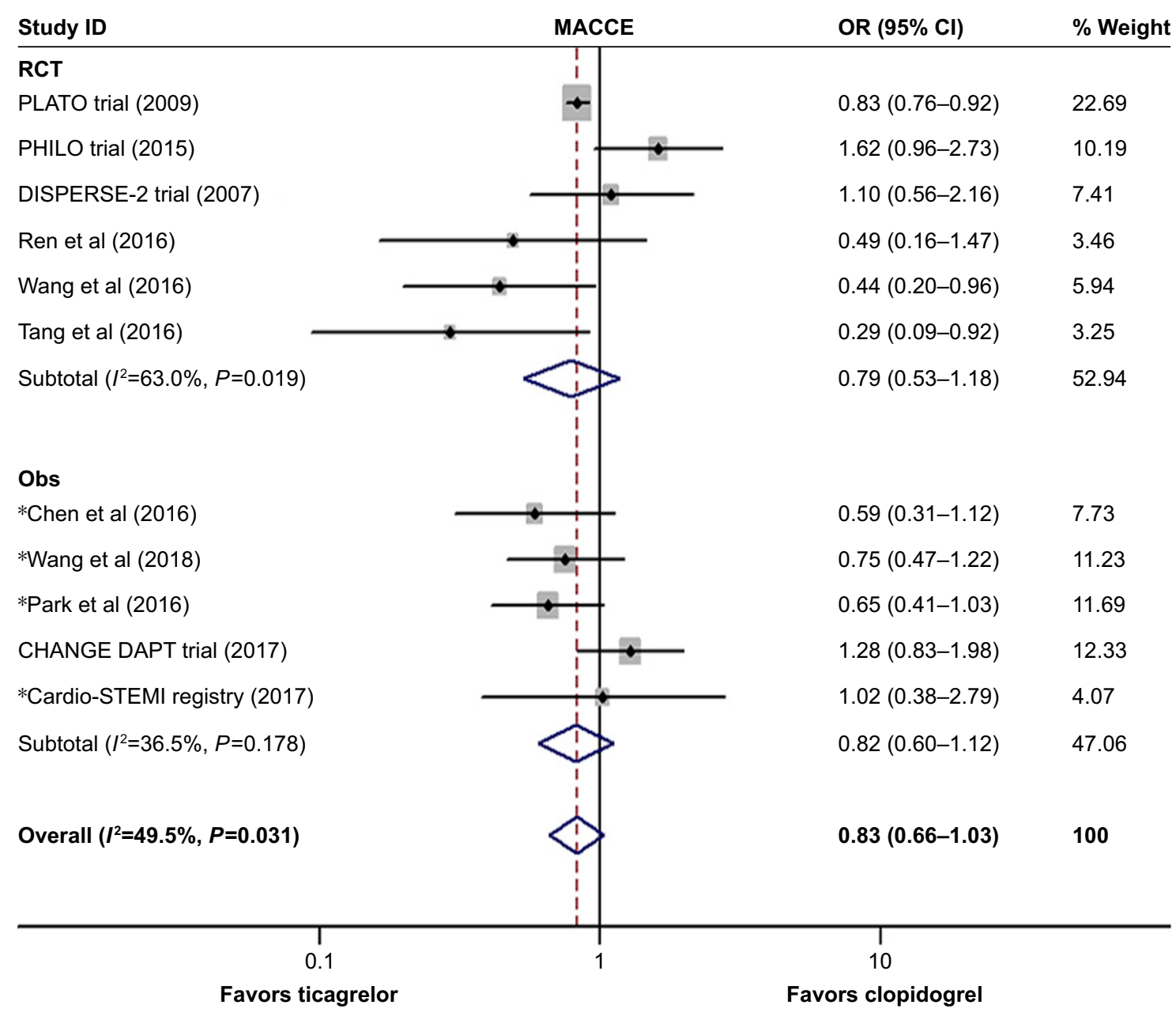

Figure 2 Forest plots of efficacy and safety end points of the included trials.

Notes: *Results following propensity-score matching. Weights are from random-effects analysis.

Abbreviations: MACCE, major adverse cardiovascular and cerebrovascular event; Obs, observational; RCT, randomized controlled trial.

\section{Myocardial infarction}

The incidence of MI did not differ significantly between the ticagrelor group and the clopidogrel group (OR 0.87, $95 \%$ CI $0.69-1.10, P=0.251 ; P^{2}=22.3 \%, P=0.244$; Figure 4$)$, or the RCT (OR 0.82, 95\% CI 0.53-1.27, $P=0.383 ; P^{2}=50.7 \%$, $P=0.088$ ) and Obs subgroups (OR 0.94, 95\% CI 0.64-1.39, $\left.P=0.762 ; I^{2}=0, P=0.594\right)$. There was no publication bias determined by Egger's test $(P=0.348)$.

\section{Stroke}

As depicted in Figure 5, there was no significant difference related to the overall risk of stroke when comparing ticagrelor to clopidogrel (OR $0.88,95 \%$ CI $0.50-1.54, P=0.653$; $\left.I^{2}=47.5 \%, P=0.055\right)$, or between the two subgroups (RCTs, OR $1.16,95 \%$ CI $0.91-1.48, P=0.243 ; I^{2}=0, P=0.488$; Obs, OR $0.66,95 \%$ CI $0.17-2.49, P=0.536 ; I^{2}=68.4 \%, P=0.023$ ). Results from Egger's test suggested no publication bias $(P=0.754)$.

\section{Definite/probable stent thrombosis}

The overall incidence of ST differed significantly between the ticagrelor group and the clopidogrel group (OR 0.74, 95\% CI 0.59-0.93, $P=0.009 ; I^{2}=0, P=0.644$; Figure 6). Analysis of the RCT group showed similarly positive results (OR 0.73, 95\% CI 0.57-0.92, $P=0.009 ; I^{2}=16.4 \%$, $P=0.274$ ), while no significant difference was obtained in the Obs subgroup (OR 0.86, 95\% CI $0.42-1.75, P=0.679$; $\left.I^{2}=0, P=0.573\right)$. Egger's test indicated no publication bias $(P=0.462)$, and the stability of the results was demonstrated by sensitivity analysis.

\section{All-cause death}

As shown in Figure 7, a lower risk of all-cause death was observed in the ticagrelor group (OR 0.81, 95\% CI 0.72-0.91, $P<0.001 ; I^{2}=16.8 \%, P=0.293$ ), which might have been driven by results from the RCT subgroup (OR $0.78,95 \%$ CI $\left.0.69-0.89, P<0.001 ; I^{2}=0.1 \%, P=0.406\right)$. There was no 


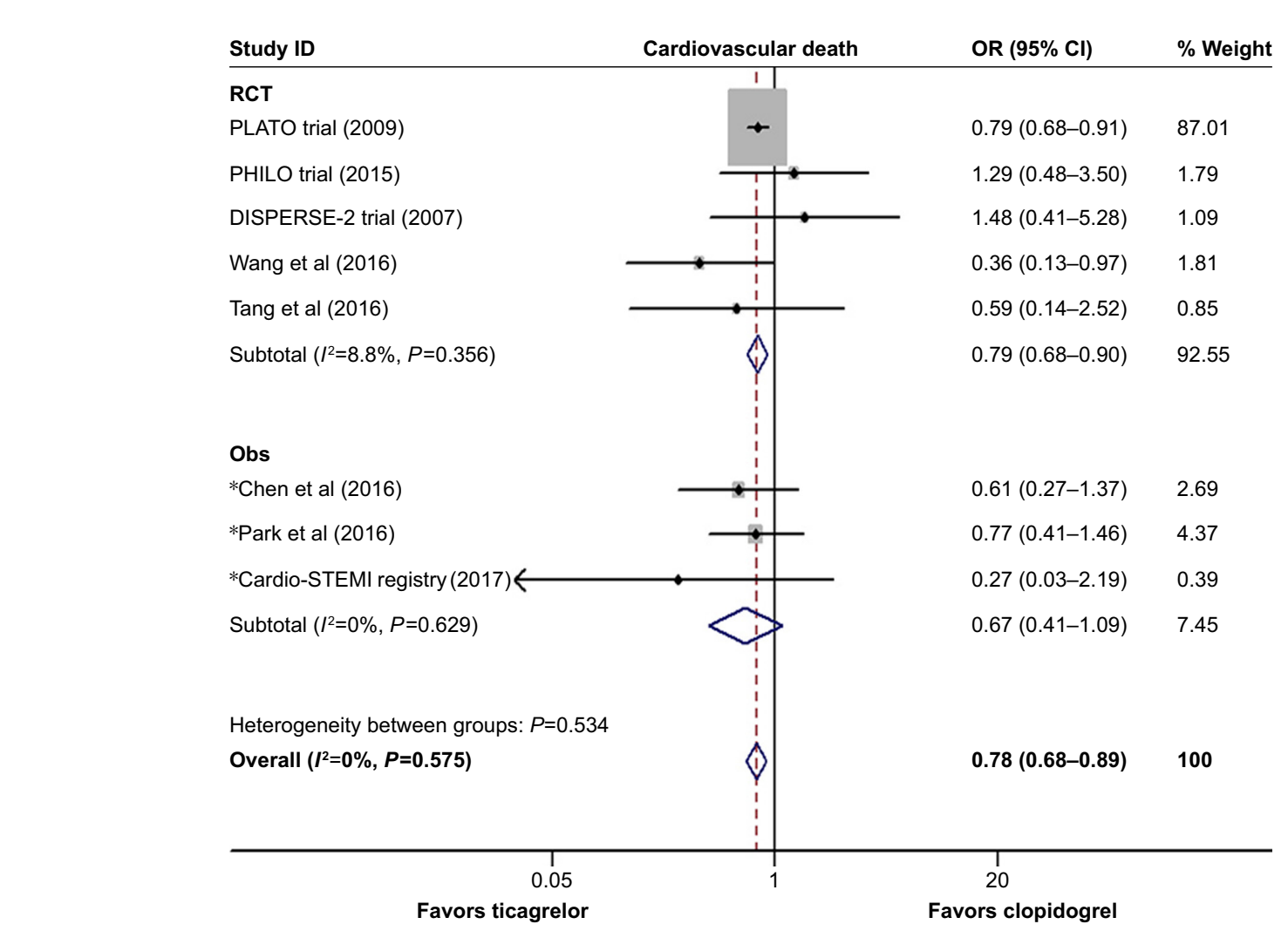

Figure 3 Cardiovascular death.

Note: *Results following propensity-score matching.

Abbreviations: Obs, observational; RCT, randomized controlled trial.

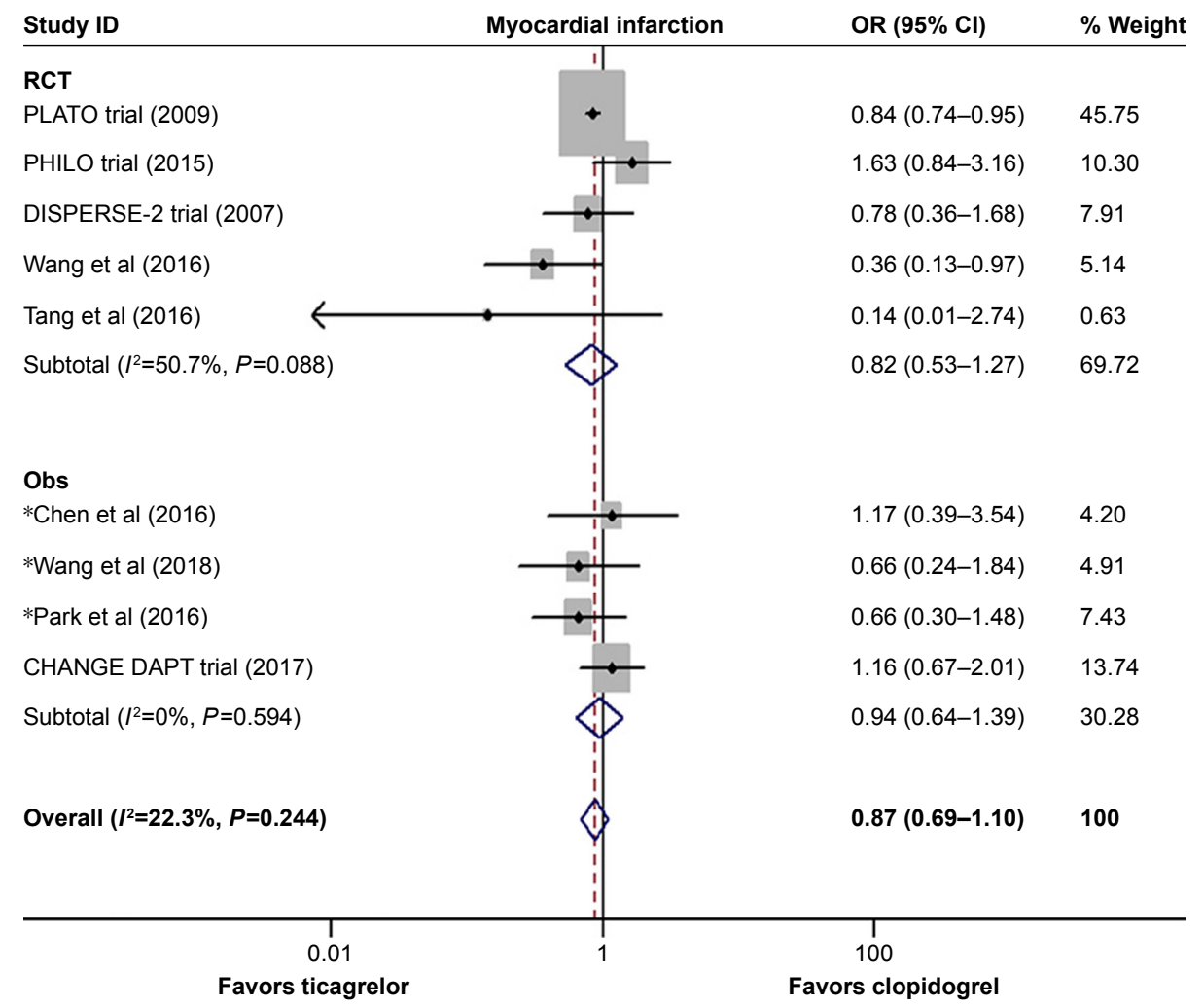

Figure 4 Myocardial infarction.

Notes: *Results following propensity-score matching. Weights are from random-effects analysis.

Abbreviations: Obs, observational; RCT, randomized controlled trial. 


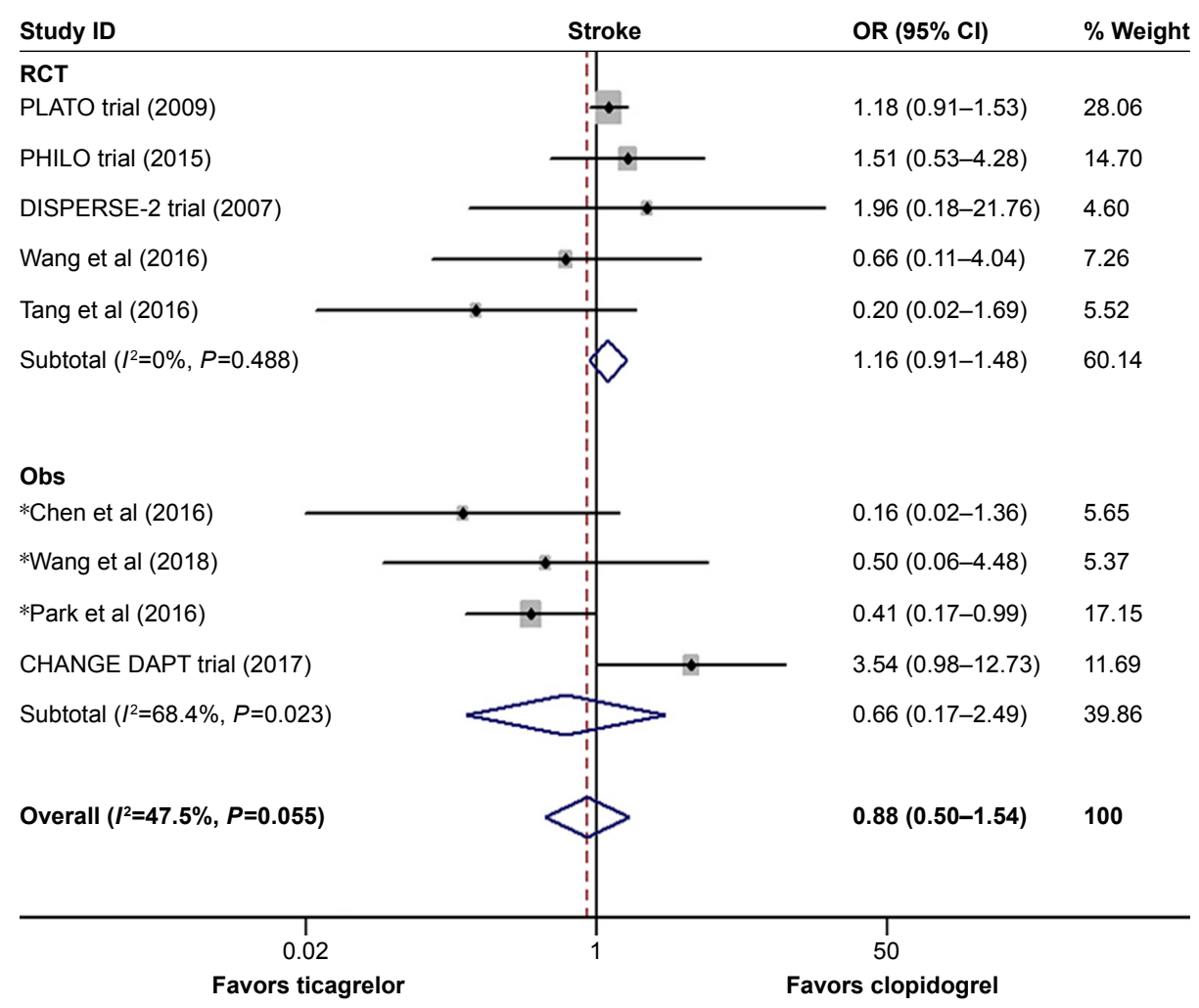

Figure 5 Stroke.

Notes: *Results following propensity-score matching. Weights are from random-effects analysis.

Abbreviations: Obs, observational; RCT, randomized controlled trial.

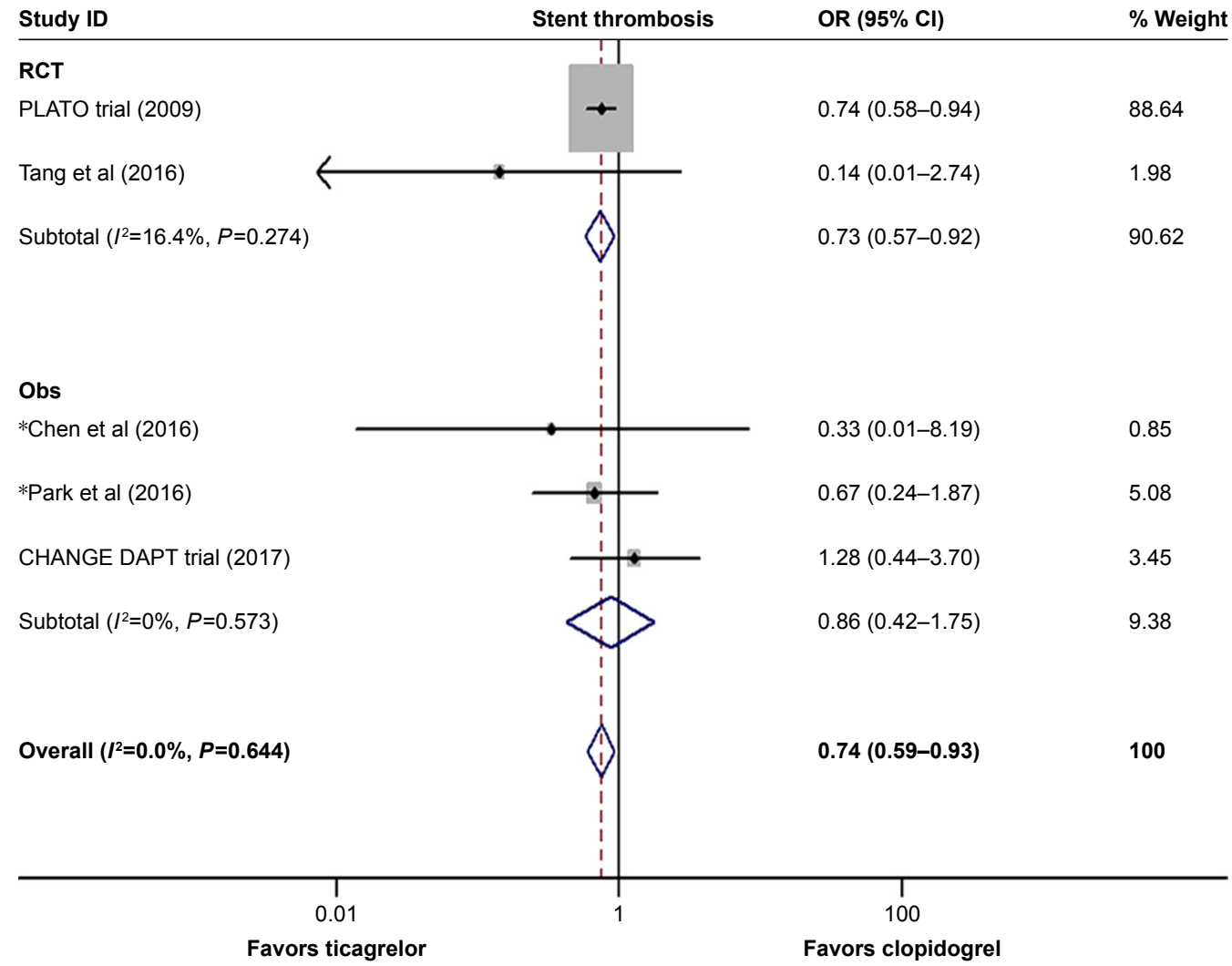

Figure 6 Stent thrombosis.

Note: *Results following propensity-score matching.

Abbreviations: Obs, observational; RCT, randomized controlled trial. 


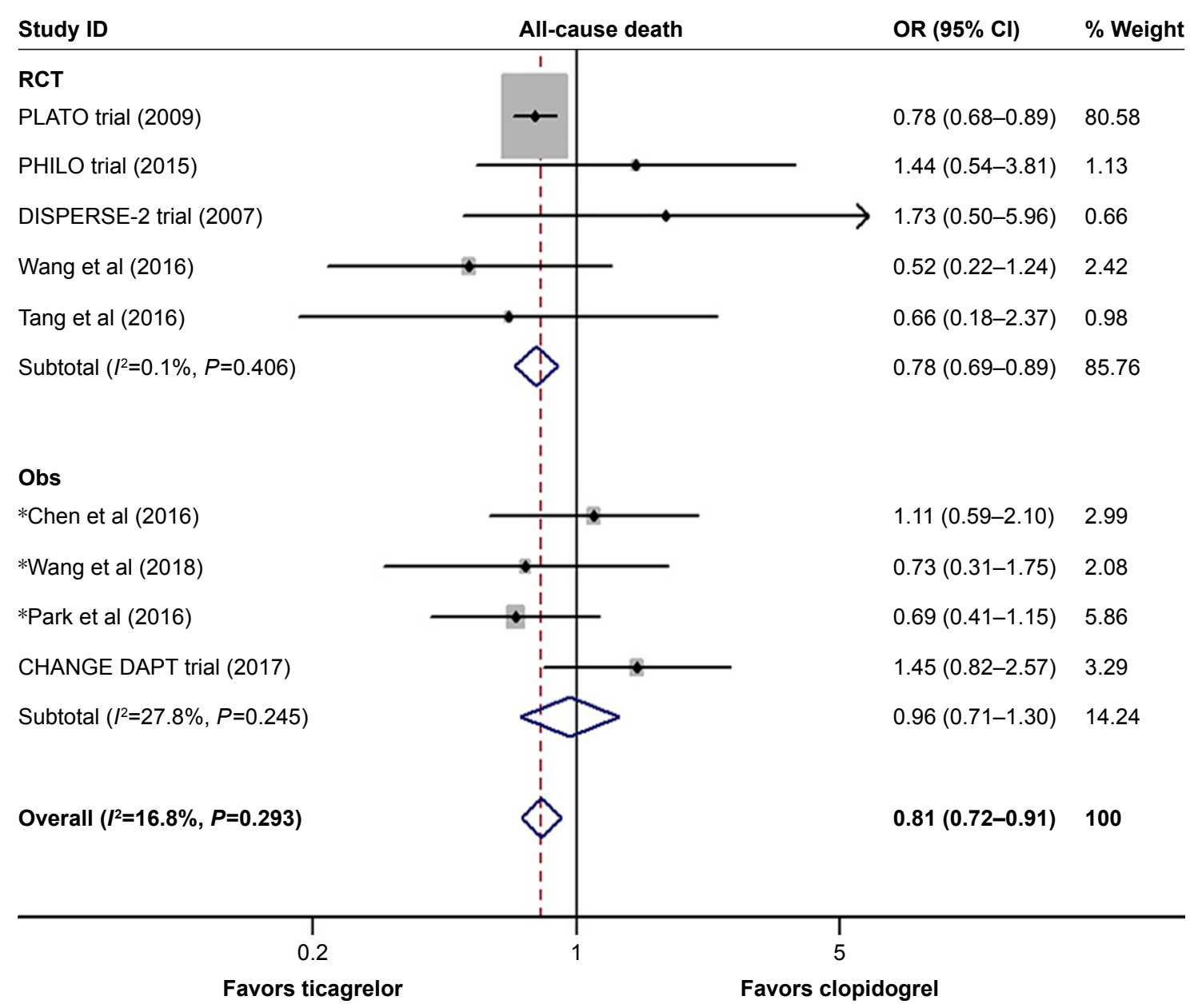

Figure 7 All-cause death.

Note: *Results following propensity-score matching.

Abbreviations: Obs, observational; RCT, randomized controlled trial.

publication bias found on Egger's test $(P=0.348)$. Sensitivity analysis proved the superior effects of ticagrelor.

\section{Safety end points}

\section{Bleeding events}

Ticagrelor significantly increased the risk of bleeding events, regardless of type: major bleeding (overall, OR 1.36, 95\% CI $1.02-1.82, P=0.038 ; P^{2}=53.7 \%, P=0.022 ; \mathrm{RCTs}$, OR $1.05,95 \%$ CI $0.94-1.18, P=0.367 ; P^{2}=1.6 \%, P=0.397 ;$ Obs, OR $1.72,95 \%$ CI 1.07-2.78, $P=0.026 ; P^{2}=44.9 \%, P=0.123$; Figure 8 ); minor bleeding (overall, OR 1.43, 95\% CI 1.25-1.63, $P<0.001$; $I^{2}=0, P=0.718$; RCTs, OR $1.40,95 \%$ CI $1.22-1.62, P<0.001$; $P^{2}=0, P=0.573$; Obs, OR $1.62,95 \%$ CI $1.10-2.40, P=0.016$; $P=0, P=0.569$; Figure 9). There was no publication bias evident ( $P=1.000,0.368$, for major and minor bleeding, respectively).

\section{Discussion}

The major finding from this meta-analysis was that ticagrelor following PCI significantly reduced the risk of cardiovascular death in patients with ACS, which might potentially lead to a reduction in the incidence of MACCEs. There were no significant reductions in incidence of MI or stroke, but both the risk of major and minor bleeding was increased significantly by ticagrelor. Also, lower occurrence of definite/probable ST and all-cause death was observed in ticagrelor group than the clopidogrel group.

Since the rapid development of interventional techniques applied widely in the clinic, improved clinical outcomes have been reported in patients with ACS.,4 Though routine DAPT (aspirin combined with clopidogrel) had been widely used following PCI, the risk of ST and restenosis were still high, leading to higher frequency of death and MI. ${ }^{5}$ The most preferred explanations for this were associated with HTPR. ${ }^{6}$ Replacement of routine clopidogrel with a new potent P2Y12-receptor antagonist (ticagrelor or prasugrel) and high maintenance doses of clopidogrel or triple antiplatelet therapy (TAPT; cilostazol adding to aspirin plus clopidogrel) were tried. In a prior randomized trial, ${ }^{27} 2,214$ patients with HTPR 


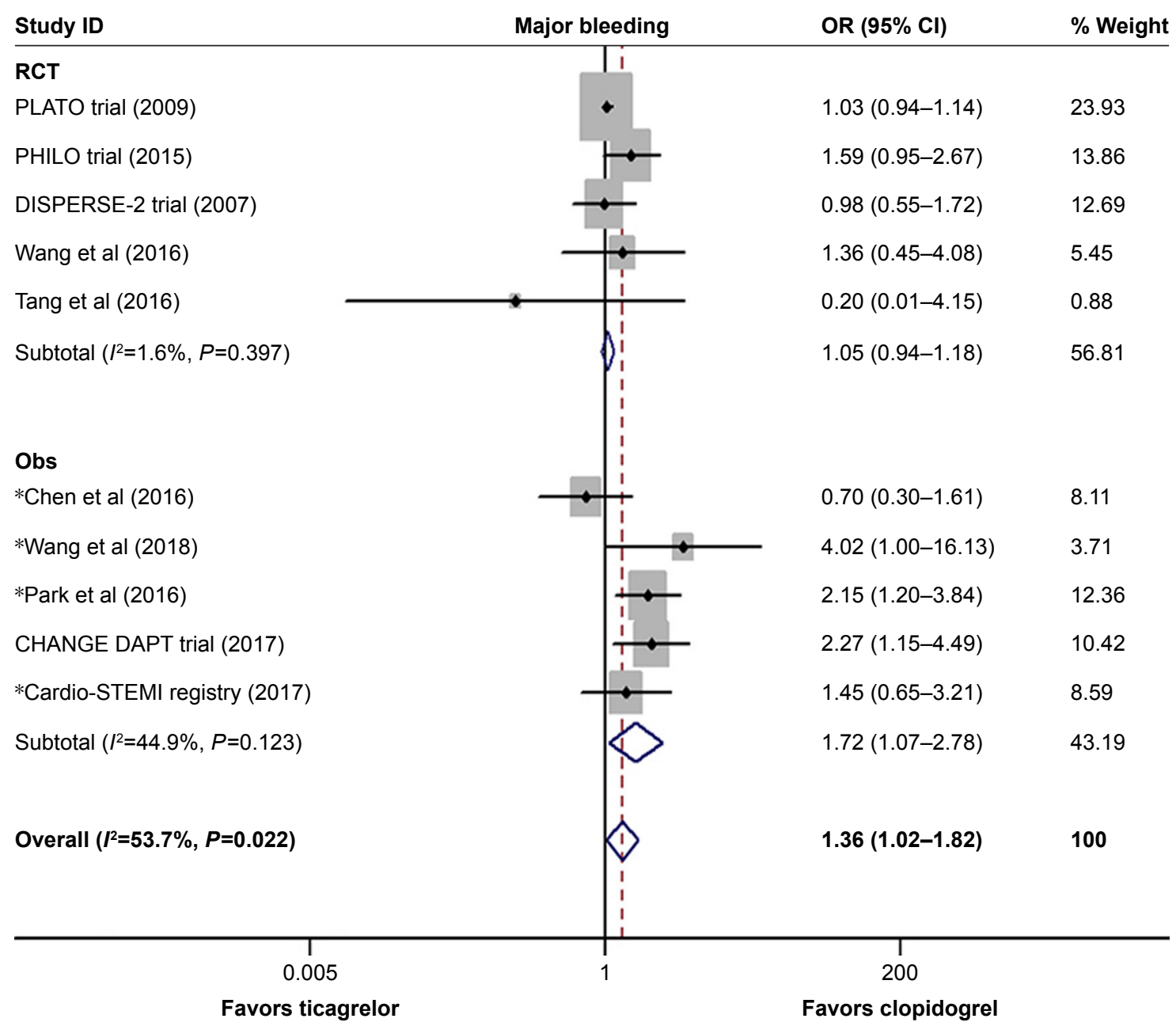

Figure 8 Major bleeding.

Notes: *Results following propensity-score matching. Weights are from random-effects analysis. Abbreviations: Obs, observational; RCT, randomized controlled trial.

were analyzed, and high-dose clopidogrel following PCI did not significantly reduce the risk of cardiac death, nonfatal MI, or ST when comparing to standard-dose clopidogrel $(2.3 \%$ vs $2.3 \%, P=0.97$ ). Additionally, a subgroup analysis from the TRILOGY ACS trial ${ }^{28}$ indicated that the occurrence of cardiovascular death, MI, or stroke among these ACS patients without revascularization did not differ significantly between the prasugrel group and the clopidogrel group at 30-month follow-up. Optimizing an antiplatelet regimen post-PCI for these patients remains a difficult problem. A previous large multicenter, double-blind randomized trial ${ }^{9}$ analyzed 18,624 patients with ACS (ticagrelor vs clopidogrel 9,333 vs 9,291) and indicated that ticagrelor was associated with a significant reduction in the risk of death from vascular causes, MI, or stroke. However, several other RCTs ${ }^{10,11}$ and Obs trials, ${ }^{12,13}$ also with large samples, demonstrated that there were no significant benefits of ticagrelor in reducing the occurrence of ischemic events among these patients receiving PCI. Furthermore, a recent meta-analysis analyzed 25,805 patients with either stable coronary artery disease or ACS and indicated that ticagrelor following PCI did not significantly reduce the incidence of all-cause mortality, major adverse cardiac events, MI, stroke, or ST, while the risk of bleeding increased instead. ${ }^{14,15}$ Another recent metaanalysis showed superiority for ticagrelor in ACS patients with chronic kidney disease without increased bleeding, but only $\sim 67.8 \%$ of included patients underwent PCI in this study. As a result, this comprehensive meta-analysis focusing on comparisons between ticagrelor and clopidogrel as part of DAPT following PCI for ACS patients was performed.

We previously performed a meta-analysis to evaluate the efficacy and safety of TAPT for patients with complex coronary lesions or suffering from ACS. The incidence of composite major adverse cardiac events (including all-cause mortality, MI, and target vessel revascularization) was lower in patients receiving TAPT rather than routine DAPT. ${ }^{29}$ 


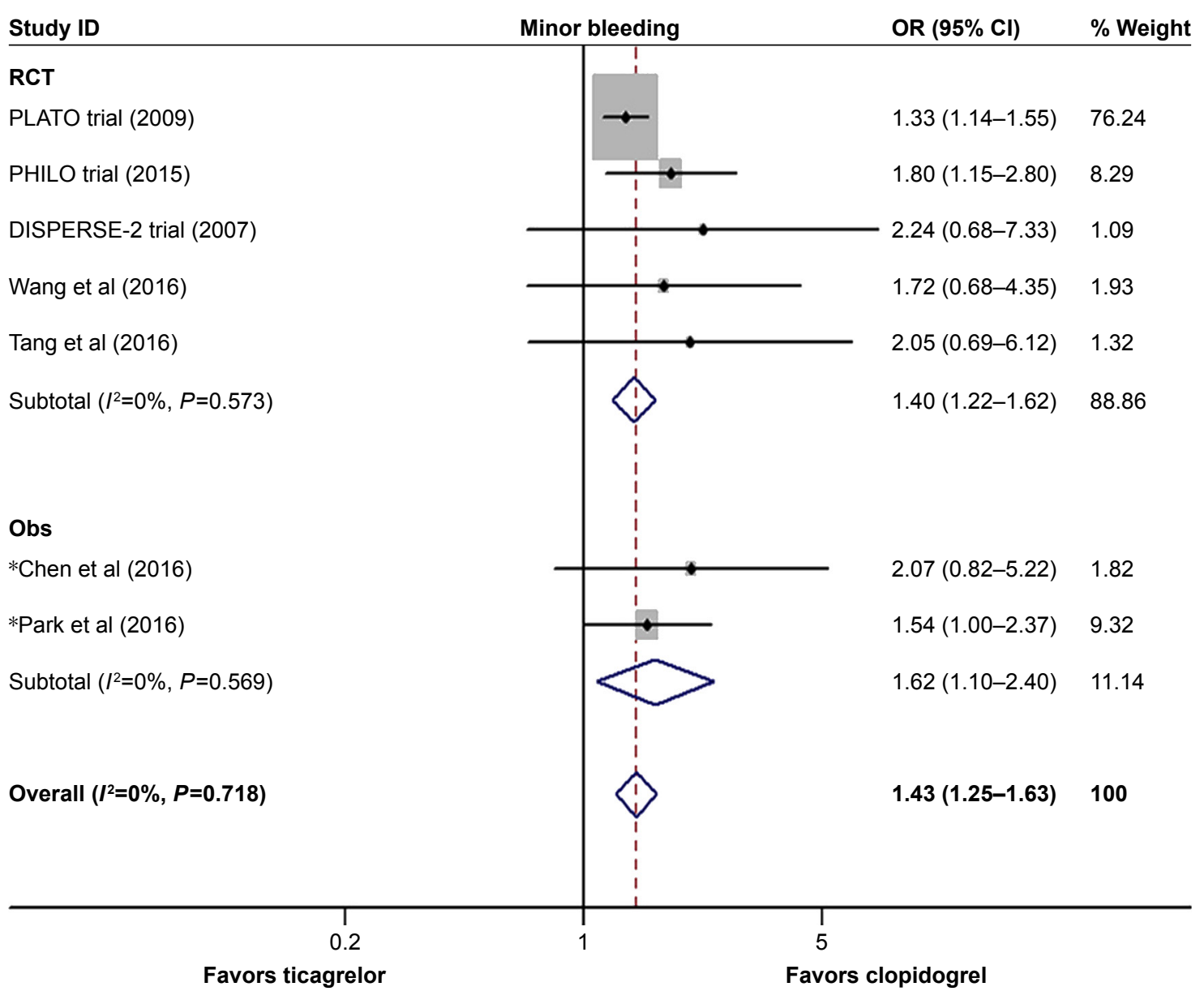

Figure 9 Minor bleeding, associated with ticagrelor compared with clopidogrel. Note: *Results following propensity-score matching.

Abbreviations: Obs, observational; RCT, randomized controlled trial.

In this present study, only a tendency toward reduction in the risk of MACCEs (composite of cardiovascular death, MI, and stroke) was observed, which might have resulted from the significantly reduced occurrence of cardiovascular death. As opposed to cilostazol, ticagrelor is a reversible and direct-acting ADP-receptor antagonist, and its main superiority manifests in faster and greater inhibitory effects on platelet aggregation than clopidogrel. ${ }^{7,8}$ Though ticagrelor similarly inhibits ADP receptors of subtype P2Y12, it acts differently mainly because of a reversible binding site, making its inhibition differ from clopidogrel. ${ }^{30}$ Cilostazol is a selective inhibitor of phosphodiesterase 3 , inhibiting platelet aggregation activated by not only ADP but also epinephrine, collagen, and arachidonic acid, which also serve as major inflammatory response factors. ${ }^{31}$ Therefore, cilostazol was thought to possess possible beneficial effects in diminishing inflammatory response. Of note, severe prothrombotic and inflammatory reactions commonly occur in the acute phase of ACS and would also be obviously ameliorated by the primary PCI procedure, especially in these patients receiving a long-time primary procedure or multiple-stent implantation. Then, platelet aggregation would not get strengthened from the severe inflammatory response during the acute phase of ACS, because it might have been suppressed by cilostazol and subsequently led to better clinical outcomes. These would be possible explanations for why only a tendency toward reduction in risk of MACCEs was acquired with respect to ticagrelor in this meta-analysis.

In addition, results from this meta-analysis demonstrated that ticagrelor can significantly decrease the incidence of definite/probable ST. To the best of our knowledge, the full expansion and apposition of implanted stents meant a successful stenting procedure, which would be much easier to achieve in simple common coronary lesions and subsequently reduce the occurrence of thrombotic events. Furthermore, the definite and potent inhibition of platelet aggregation associated with ticagrelor ${ }^{30}$ would also play a critical role during the process of endothelialization, considered beneficial 
to prevent restenosis in implanted stents. Based on these potential factors, the incidence of cardiovascular death in the ticagrelor group decreased significantly. Simultaneously, the risk of bleeding, regardless of type, increased significantly in the ticagrelor group in our study, in contrast to the results in the PLATO trial $(11.6 \%$ vs $11.2 \%, P=0.43) .{ }^{9}$ Possible reasons were thought to be related not only to the definite and potent inhibition of platelet aggregation with respect to ticagrelor itself but also the higher proportion of Asian patients (28.6\%) in this meta-analysis, while there were only $\sim 5.9 \%$ Asians in the PLATO trial instead. It is well known that Asian patients commonly present lower body-mass index values, whereby bleeding might occur more easily under the same dose of ticagrelor. On the other hand, PCI was performed with a radial or femoral approach in these patients and also played as a key role in influencing the final safety results. In the 2018 ESC/EACTS guidelines, radial access was strongly recommended as a standard approach for coronary angiography and PCI (Class IA), due mainly to the significantly reduced incidence of procedure-related major bleeding. ${ }^{32}$

\section{Limitations}

There still several limitations that should be acknowledged in the current meta-analysis. First, no individual patient data were analyzed and several RCTs had small samples, which might have influenced the final results. Second, accurate details of the PCI procedures were absent from our paper, due mainly to the inadequacy of relevant data, including time of procedure, types of implanted stents, selection of different two-stent techniques for potentially occurred bifurcation lesions, or choice of sheaths of different sizes. Third, there were no definite maintained durations or dosage of ticagrelor for these patients and no limited follow-up in these trials, which might also have swayed the final results. Forth, possible drug adverse reactions reduced tolerance and induced discontinuation of ticagrelor would also be unavoidable interfering factors.

\section{Conclusion}

Though only a tendency toward reduction in the risk of MACCEs was observed, ticagrelor as part of DAPT following PCI still seemed to be superior to clopidogrel for ACS patients, mainly because of the significantly decreased incidence of cardiovascular death and ST. However, increased occurrence of bleeding was also observed, and safety might need to be further evaluated. More powerful relevant randomized trials are still warranted to guide clinical decision-making in this area.

\section{Disclosure}

The authors report no conflicts of interest in this work.

\section{References}

1. Goldberg RJ, Spencer FA, Gore JM, Lessard D, Yarzebski J. Thirty-year trends (1975 to 2005) in the magnitude of, management of, and hospital death rates associated with cardiogenic shock in patients with acute myocardial infarction: a population-based perspective. Circulation. 2009;119(9):1211-1219.

2. O'Gara PT, Kushner FG, Ascheim DD, et al; Foundation/American Heart Association Task Force on Practice Guidelines. 2013 ACCF/AHA guideline for the management of ST-elevation myocardial infarction: a report of the American College of cardiology Foundation/American Heart Association Task Force on practice guidelines. J Am Coll Cardiol. 2013;61(4):e78-e140.

3. Neumann FJ, Sousa-Uva M, Ahlsson A, et al; ESC Scientific Document Group. ESC/EACTS guidelines on myocardial revascularization. Eur Heart J. 2018;25:2018.

4. Cowley MJ, Kuritzky L. Developments in antiplatelet therapy for acute coronary syndromes and considerations for long-term management Curr Med Res Opin. 2009;25(6):1477-1490.

5. Grove EL, Würtz M, Thomas MR, Kristensen SD. Antiplatelet therapy in acute coronary syndromes. Expert Opin Pharmacother. 2015;16(14) 2133-2147.

6. Parodi G, Marcucci R, Valenti R, et al. High residual platelet reactivity after clopidogrel loading and long-term cardiovascular events among patients with acute coronary syndromes undergoing PCI. JAMA. 2011; 306(11):1215-1223

7. Storey RF, Husted S, Harrington RA, et al. Inhibition of platelet aggregation by AZD6140, a reversible oral P2Y12 receptor antagonist, compared with clopidogrel in patients with acute coronary syndromes. J Am Coll Cardiol. 2007;50(19):1852-1856.

8. Husted S, Emanuelsson H, Heptinstall S, Sandset PM, Wickens M, Peters G. Pharmacodynamics, pharmacokinetics, and safety of the oral reversible P2Y12 antagonist AZD6140 with aspirin in patients with atherosclerosis: a double-blind comparison to clopidogrel with aspirin. Eur Heart J. 2006;27(9):1038-1047.

9. Wallentin L, Becker RC, Budaj A, et al; DISPERSE-2 Investigators Ticagrelor versus clopidogrel in patients with acute coronary syndromes. N Engl J Med. 2009;361(11):1045-1057.

10. Cannon CP, Husted S, Harrington RA, et al. Safety, tolerability, and initial efficacy of AZD6140, the first reversible oral adenosine diphosphate receptor antagonist, compared with clopidogrel, in patients with non-st-segment elevation acute coronary syndrome. J Am Coll Cardiol. 2007;50(19):1844-1851.

11. Goto S, Huang CH, Park SJ, Emanuelsson H, Kimura T. Ticagrelor vs. Clopidogrel in Japanese, Korean and Taiwanese patients with acute coronary syndrome-randomized, double-blind, Phase III PHILO study. Circ J. 2015;79(11):2452-2460.

12. Zocca P, van der Heijden LC, Kok MM, et al. Clopidogrel or ticagrelor in acute coronary syndrome patients treated with newer-generation drugeluting stents: change DAPT. Euro Intervention. 2017;13(10):1168-1176.

13. Park KH, Jeong MH, Ahn Y, et al; KAMIR-NIH registry investigators Comparison of short-term clinical outcomes between ticagrelor versus clopidogrel in patients with acute myocardial infarction undergoing successful revascularization; from Korea acute myocardial infarction Registry - National Institute of health. Int J Cardiol. 2016;215: 193-200.

14. Guan W, Lu H, Yang K. Choosing between ticagrelor and clopidogrel following percutaneous coronary intervention: a systematic review and meta-analysis (2007-2017). Medicine (Baltimore). 2018;97(43):e12978.

15. Bonello L, Laine M, Lemesle G, et al. Meta-analysis of potent P2Y12-ADP receptor antagonist therapy compared to clopidogrel therapy in acute coronary syndrome patients with chronic kidney disease. Thromb Haemost. 2018;118(10):1839-1846. 
16. Jadad AR, Moore RA, Carroll D, et al. Assessing the quality of reports of randomized clinical trials: is blinding necessary? Control Clin Trials. 1996;17(1):1-12.

17. Biondi-Zoccai GG, Abbate A, Agostoni P, et al. Long-term benefits of an early invasive management in acute coronary syndromes depend on intracoronary stenting and aggressive antiplatelet treatment: a metaregression. Am Heart J. 2005;149(3):504-511.

18. Ren Q, Ren C, Liu X, Dong C, Zhang X. Ticagrelor vs. clopidogrel in non-ST-elevation acute coronary syndromes. Herz. 2016;41(3):246-249.

19. Wang HY, Li Y, Xu XM, Li J, Han YL. Impact of baseline bleeding risk on efficacy and safety of ticagrelor versus clopidogrel in Chinese patients with acute coronary syndrome undergoing percutaneous coronary intervention. Chin Med J (Engl). 2018;131(17):2017-2024.

20. Mauri L, Hsieh WH, Massaro JM, Ho KK, D’Agostino R, Cutlip DE. Stent thrombosis in randomized clinical trials of drug-eluting stents. N Engl J Med. 2007;356(10):1020-1029.

21. Liberati A, Altman DG, Tetzlaff J, et al. The PRISMA statement for reporting systematic reviews and meta-analyses of studies that evaluate healthcare interventions: explanation and elaboration. BMJ. 2009;339:b2700.

22. Egger M, Davey Smith G, Schneider M, Minder C. Bias in metaanalysis detected by a simple, graphical test. BMJ. 1997;315(7109): 629-634.

23. Wang H, Wang X. Efficacy and safety outcomes of ticagrelor compared with clopidogrel in elderly Chinese patients with acute coronary syndrome. Ther Clin Risk Manag. 2016;12:1101-1105.

24. Tang X, Li R, Jing Q, et al. Assessment of ticagrelor versus clopidogrel treatment in patients with ST-elevation myocardial infarction undergoing primary percutaneous coronary intervention. J Cardiovasc Pharmacol. 2016;68(2):115-120.
25. Chen IC, Lee CH, Fang CC, et al. Efficacy and safety of ticagrelor versus clopidogrel in acute coronary syndrome in Taiwan: a multicenter retrospective pilot study. J Chin Med Assoc. 2016;79(10):521-530.

26. Vercellino M, Sànchez FA, Boasi V, et al. Ticagrelor versus clopidogrel in real-world patients with ST elevation myocardial infarction: 1-year results by propensity score analysis. BMC Cardiovasc Disord. 2017;17(1):97.

27. Price MJ, Berger PB, Teirstein PS, et al. Standard- vs high-dose clopidogrel based on platelet function testing after percutaneous coronary intervention: the GRAVITAS randomized trial. JAMA. 2011;305(11): 1097-1105.

28. Gurbel PA, Erlinge D, Ohman EM, et al. Platelet function during extended prasugrel and clopidogrel therapy for patients with ACS treated without revascularization: the trilogy ACS platelet function substudy. JAMA. 2012;308(17):1785-1794.

29. Fan ZG, Ding GB, Li XB, Gao XF, Gao YL, Tian NL. The clinical outcomes of triple antiplatelet therapy versus dual antiplatelet therapy for high-risk patients after coronary stent implantation: a meta-analysis of 11 clinical trials and 9,553 patients. Drug Des Devel Ther. 2016;10:3435-3448.

30. Birkeland K, Parra D, Rosenstein R. Antiplatelet therapy in acute coronary syndromes: focus on ticagrelor. J Blood Med. 2010;1:197-219.

31. Cone J, Wang S, Tandon N, et al. Comparison of the effects of cilostazol and milrinone on intracellular cAMP levels and cellular function in platelets and cardiac cells. J Cardiovasc Pharmacol. 1999; 34(4):497-504.

32. Valgimigli M, Gagnor A, Calabró P, et al; MATRIX Investigators. Radial versus femoral access in patients with acute coronary syndromes undergoing invasive management: a randomised multicentre trial. Lancet. 2015;385(9986):2465-2476.
Drug Design, Development and Therapy

\section{Publish your work in this journal}

Drug Design, Development and Therapy is an international, peerreviewed open-access journal that spans the spectrum of drug design and development through to clinical applications. Clinical outcomes, patient safety, and programs for the development and effective, safe, and sustained use of medicines are the features of the journal, which

\section{Dovepress}

has also been accepted for indexing on PubMed Central. The manuscript management system is completely online and includes a very quick and fair peer-review system, which is all easy to use. Visit http://www.dovepress.com/testimonials.php to read real quotes from published authors. 